\title{
Computational Simulation of the Impact of System Perturbation on Stabilization of the Growth of Two Political Parties
}

\section{*1BAZUAYE FRANK ETIN-OSA}

\author{
Department of mathematics and statistics, university of Port Harcourt, \\ Port Harcourt, Rivers State \\ Email: febazuave@yahoo.com
}

\begin{abstract}
Qualitative characterization of the perturbation of the growth of two political parties which in nature requires a strong numerical tool for its analysis. We have therefore in this study utilized a Matlab standard solver for ordinary differential equations ODE 45to investigate the impact of system perturbation otherwise called random fluctuation on the stabilization of two interacting political parties in a developing democracy and to evaluate the qualitative characterization of interacting political parties due to $0.01,0.10,5.00$ and 10.00 random noise system perturbation. The result indicates that as the system perturbation increases, the level of de-stabilization of the entire political system increases. This research has re-enforced the impact of de-stabilization factors such as lack of internal democracy in political parties has on the de-stabilization of political parties in a developing democracy and if avoided, will lead to a robust and growth of parties in developing democracy. (C) JASEM https://dx.doi.org/10.4314/jasem.v21i2.21
\end{abstract}

Keywords: System perturbation, de-stabilization, political parties, stabilization, qualitative characterization.

Addressing the challenges of de-stabilization, it is important to investigate the impact of system perturbation driving factors such as failure in election, cross carpeting by political gladiators, political instability, etc can actually affect the potential and motivation of people in the political activities and bring about a non- participatory democracy As it can see in (Huckfeldt and Kohfeld,1992).Although, we have agreed that in a deterministic sense, the parameterization of (Arvhind
,2012) steady state solution is stable. However, what is the extent of the per capital recruitment rate $\eta_{1}$ of party $Q$ and the per capital recruitment rate $\eta_{2}$ of party $R$ system perturbation has on the stabilization? This is a neglected aspect of modeling two interacting political parties, which remains an open research question since the activities of political parties are dynamical in nature,(Arato,2003),

Mathematical Formulation: Following Arvind (2012), the modeling equation which is a dynamical system ,is given by

$$
\begin{aligned}
& \frac{d P}{d t}=\varphi m-\eta_{1} P \frac{Q}{M}-\eta_{2} P \frac{R}{M}-\varphi P \\
& \frac{d Q}{d t}=\eta_{1} P \frac{Q}{M}-\gamma_{1} Q \frac{R}{M}-\gamma_{2} R \frac{Q}{M}-\varphi Q \\
& \frac{d R}{d t}=\eta_{2} P \frac{R}{M}+\gamma_{1} Q \frac{R}{M}-\gamma_{2} R \frac{Q}{M}-\varphi R
\end{aligned}
$$

With the conditions the initial conditions $P(0)>0, Q(0) \geq 0, \quad R(0) \geq 0$.

At the steady state solution $\frac{d M}{d t}=0$

This is an extended Lotka-Volterra multi-substitution model as in (Morris and David 2003).

Method of Solution: The numerical simulation we are proposing for the solution of this complex class of problem (1) is called the Matlab Numerical simulation software. The philosophy behind this method is to know the impact of varying the level of perturbation on the solution trajectory values. 
Following (Arvind, 2012) and (Ekaka-a, 2009), we consider the following precise deterministic parameter values;

$$
\eta_{1}=0.0417, \eta_{2}=0.0278, \gamma_{1}=0.0236, \gamma_{2}=0.0097
$$

The major method of analysis is based on the implementation of the MATLAB ODE 45 numerical scheme which is a Robust Runge-kutta scheme and evaluates the qualitative characterization of interacting political parties due to $0.01,0.1,1,5.0$ and 10.0 random noises and observes the qualitative behavior on the solution trajectories.

The full results of applying this method are presented and discussed next.

\section{RESULTS AND DISCUSSION}

It can be observed from Table 1 to Table 5 that as the system perturbation increases, the level of destabilization of the entire system increases. So, it is cleared from this novel contribution that as the independent variable $t$ tends to infinity, the solution trajectory due to a random system perturbation generally outweighs the solution trajectory without a random system perturbation. On the basis of this systematic analysis, we have observed that a random noise system perturbation has the potential to destabilize the deterministic dynamical system that describes the interaction between two political parties in developing democracies like Nigeria.

Table 1: Evaluating the qualitative characterization of interacting political parties due to 0.01 random noise system perturbation using ODE 45

\begin{tabular}{cccccc}
\hline Example & RS & $\eta_{1}(4)$ & $\eta_{1} r n$ & $\eta_{2}(4)$ & $\eta_{2} r n$ \\
\hline $\mathbf{1}$ & 1 & 1.43651711325650 & 1.0609576567136 & 10.7980529152492 & 2.48821529715523 \\
$\mathbf{2}$ & 2 & 1.43651711325650 & 1.05790549566056 & 10.7980529152492 & 2.48781481084994 \\
$\mathbf{3}$ & 3 & 1.43651711325650 & 1.06625404474447 & 10.7980529152492 & 2.49029570275644 \\
$\mathbf{4}$ & 4 & 1.43651711325650 & 1.05680699689741 & 10.7980529152492 & 2.48828934088830 \\
$\mathbf{5}$ & 5 & 1.43651711325650 & 1.05991760322433 & 10.7980529152492 & 2.48305162621826 \\
$\mathbf{6}$ & 6 & 1.43651711325650 & 1.06133475898536 & 10.7980529152492 & 2.48289274320012 \\
$\mathbf{7}$ & 7 & 1.43651711325650 & 1.06085914104013 & 10.7980529152492 & 2.48365162051966 \\
$\mathbf{8}$ & 8 & 1.43651711325650 & 1.06127674399038 & 10.7980529152492 & 2.48701821842759 \\
$\mathbf{9}$ & 9 & 1.43651711325650 & 1.05824156275381 & 10.7980529152492 & 2.48131002946453 \\
$\mathbf{1 0}$ & 10 & 1.43651711325650 & 1.05739994984151 & 10.7980529152492 & 2.48395500010629 \\
\hline
\end{tabular}

When the value of random noise perturbation is 0.01 , the numerical simulation random noise value ranges from the value of 1.05824156275381 to 1.06625404474447 and 2.48131002946453 to 2.49029570275644 for the intrinsic growth rates $\eta_{1} r n$ and $\eta_{2} r n$ respectively of the interacting two political parties.

Table 2: Evaluating the qualitative characterization of interacting political parties due to 0.1 random noise system perturbation using ODE 45

\begin{tabular}{cccccc}
\hline Example & $\mathbf{R S}$ & $\eta_{1}(4)$ & $\eta_{1} r n$ & $\eta_{2}(4)$ & $\eta_{2} r n$ \\
\hline $\mathbf{1}$ & 1 & 1.43651711325650 & 1.2278527712168 & 10.7980529152492 & 2.62400631245802 \\
$\mathbf{2}$ & 2 & 1.43651711325650 & 1.17524014827114 & 10.7980529152492 & 2.69697672824029 \\
$\mathbf{3}$ & 3 & 1.43651711325650 & 1.25026500565991 & 10.7980529152492 & 2.63107198302351 \\
$\mathbf{4}$ & 4 & 1.43651711325650 & 1.22984058004148 & 10.7980529152492 & 2.69833190689295 \\
$\mathbf{5}$ & 5 & 1.43651711325650 & 1.21890869254285 & 10.7980529152492 & 2.65872818385740 \\
$\mathbf{6}$ & 6 & 1.43651711325650 & 1.18967112729009 & 10.7980529152492 & 2.67038812676433 \\
$\mathbf{7}$ & 7 & 1.43651711325650 & 1.19737145125192 & 10.7980529152492 & 2.69471287056911 \\
$\mathbf{8}$ & 8 & 1.43651711325650 & 1.16667083134789 & 10.7980529152492 & 2.70316472749864 \\
$\mathbf{9}$ & 9 & 1.43651711325650 & 1.21272231323964 & 10.7980529152492 & 2.68372387701938 \\
$\mathbf{1 0}$ & 10 & 1.43651711325650 & 1.16461465308184 & 10.7980529152492 & 2.71849117110819 \\
\hline
\end{tabular}

When the value of random noise perturbation is 0.1 , the numerical simulation random noise value ranges from the value of 1.16461465308184 to 1.25026500565991 and 2.62400631245802to 2.71849117110819 for the intrinsic growth rates $\eta_{1} r n$ and $\eta_{2} r n$ respectively of the interacting two political parties. 
Table 3: Evaluating the qualitative characterization of interacting political parties due to 1.0 random noise system perturbation using ODE45

\begin{tabular}{cccccc}
\hline Example & RS & $\eta_{1}(4)$ & $\eta_{1} r n$ & $\eta_{2}(4)$ & $\eta_{2} r n$ \\
\hline $\mathbf{1}$ & 1 & 1.43651711325650 & 2.5462969084112 & 10.7980529152492 & 4.48334765667066 \\
$\mathbf{2}$ & 2 & 1.43651711325650 & 2.5374269585154 & 10.7980529152492 & 4.41568117876753 \\
$\mathbf{3}$ & 3 & 1.43651711325650 & 2.5802537978094 & 10.7980529152492 & 4.59931999407648 \\
$\mathbf{4}$ & 4 & 1.43651711325650 & 2.4309617589688 & 10.7980529152492 & 4.56505670388324 \\
$\mathbf{5}$ & 5 & 1.43651711325650 & 2.5053024168849 & 10.7980529152492 & 4.57407583642139 \\
$\mathbf{6}$ & 6 & 1.43651711325650 & 2.6270813291340 & 10.7980529152492 & 4.76683233496164 \\
$\mathbf{7}$ & 7 & 1.43651711325650 & 2.5189851694690 & 10.7980529152492 & 4.55790894565828 \\
$\mathbf{8}$ & 8 & 1.43651711325650 & 2.5644207874087 & 10.7980529152492 & 4.48189851234733 \\
$\mathbf{9}$ & 9 & 1.43651711325650 & 2.3973594428491 & 10.7980529152492 & 4.44695160895281 \\
$\mathbf{1 0}$ & 10 & 1.43651711325650 & 2.5133940503448 & 10.7980529152492 & 4.58050523700687 \\
\hline
\end{tabular}

Similarly, when the value of random noise perturbation is 1.0 , the numerical simulation

Random noise value ranges from the value of 2.3973594428491 to 2.6270813291340 and

4.41568117876753 to4.76683233496164 for the intrinsic growth rates $\eta_{1} r n$ and $\eta_{2} r n$ respectively of the interacting two political parties.

Table 4: Evaluating the qualitative characterization of interacting political parties due to 5.0 random noise system perturbation using ODE45

\begin{tabular}{|c|c|c|c|c|c|}
\hline Example & $\mathbf{R S}$ & $\eta_{1}(4)$ & $\eta_{1} r n$ & $\eta_{2}(4)$ & $\eta_{2} r n$ \\
\hline 1 & 1 & 1.43651711325650 & 6.3016067016574 & 10.7980529152492 & 13.3789554796706 \\
\hline 2 & 2 & 1.43651711325650 & 6.378955479670 & 10.7980529152492 & 13.2439302145988 \\
\hline 3 & 3 & 1.43651711325650 & 6.7633452903934 & 10.7980529152492 & 13.4420359648161 \\
\hline 4 & 4 & 1.43651711325650 & 6.5814343554167 & 10.7980529152492 & 14.2039463727159 \\
\hline 5 & 5 & 1.43651711325650 & 6.6064496058526 & 10.7980529152492 & 14.3230790042630 \\
\hline 6 & 6 & 1.43651711325650 & 6.4026092488267 & 10.7980529152492 & 14.6951902151518 \\
\hline 7 & 7 & 1.43651711325650 & 6.6843117554080 & 10.7980529152492 & 13.0967679462801 \\
\hline 8 & 8 & 1.43651711325650 & 6.6336612871534 & 10.7980529152492 & 13.1949554862764 \\
\hline 9 & 9 & 1.43651711325650 & 6.8926304345713 & 10.7980529152492 & 14.1384515328078 \\
\hline 10 & 10 & 1.43651711325650 & 6.8509580132006 & 10.7980529152492 & 14.2899380729331 \\
\hline
\end{tabular}

When the value of random noise perturbation is 5.0, the numerical simulation

Random noise value ranges from the value of 6.3016067016574 to 6.8926304345713 and

13.1949554862764 to14.6951902151518 for the intrinsic growth rates $\eta_{1} r n$ and $\eta_{2} r n$ respectively of the interacting two political parties.

Table 5: Evaluating the qualitative characterization of interacting political parties due to 10.0 random noise system perturbation using ODE45

\begin{tabular}{cccccc}
\hline Example & $\mathbf{R S}$ & $\eta_{1}(4)$ & $\eta_{1} r n$ & $\eta_{2}(4)$ & $\eta_{2} r n$ \\
\hline $\mathbf{1}$ & 1 & 1.43651711325650 & 8.8840468198663 & 10.7980529152492 & 26.9016524675767 \\
$\mathbf{2}$ & 2 & 1.43651711325650 & 9.3016792483179 & 10.7980529152492 & 26.4605476780696 \\
$\mathbf{3}$ & 3 & 1.43651711325650 & 8.8141689814512 & 10.7980529152492 & 25.5261725024278 \\
$\mathbf{4}$ & 4 & 1.43651711325650 & 9.9403227931174 & 10.7980529152492 & 25.6636555375322 \\
$\mathbf{5}$ & 5 & 1.43651711325650 & 9.5961209073984 & 10.7980529152492 & 25.9218805979897 \\
$\mathbf{6}$ & 6 & 1.43651711325650 & 8.9128463048855 & 10.7980529152492 & 25.5802604455235 \\
$\mathbf{7}$ & 7 & 1.43651711325650 & 9.2914952824882 & 10.7980529152492 & 25.7037420305581 \\
$\mathbf{8}$ & 8 & 1.43651711325650 & 9.3784420158988 & 10.7980529152492 & 26.7615214099693 \\
$\mathbf{9}$ & 9 & 1.43651711325650 & 9.3819929912604 & 10.7980529152492 & 27.8023554675845 \\
$\mathbf{1 0}$ & 10 & 1.43651711325650 & 9.8893213894481 & 10.7980529152492 & 25.1545358969362 \\
\hline
\end{tabular}

Lastly, when the value of random noise perturbation is 10.0, the numerical simulation

Random noise value ranges from the value of 8.8141689814512 to9.9403227931174 and

25.1545358969362 to 27.8023554675845 for the intrinsic growth rates $\eta_{1} r n$ and $\eta_{2} r n$ respectively of the interacting two political parties. 
From the analysis carried out, we would recommend a reduction in the factors that bring about fluctuation and cause de-stabilization. For example, lack of internal democracy, fail electoral promises, imposition of candidates, can have negative implications in the growth and survival of political parties

Conclusion: This paper has presented a novel contribution to knowledge by successfully utilizing numerical simulation technique to re-enforce the fact that a reduction in the factors that cause the destabilization of survival of political parties in a developing democracy, will lead to a robust and growth of parties.

\section{REFERENCES}

Arvind K.M (2012), A simple mathematical model for the spread of two political parties. Nonlinear analysis modeling and control 17(3), 343-354.
Arato, M. (2003), A famous nonlinear stochastic equation; Lotka-Volterra model with diffusion. Mathematical and computer modeling, 38(7/9), 709-726.

Ekaka-a E.N. (2009), Computational and Mathematical modeling of plant species interactions in a harsh climate. Ph.D Thesis, Department of Mathematics, the University of Liverpool and the University of Chester, United Kingdom.

Huckfeldt, C.W, Kohfeld. (1992), Electoral stability and the decline of class in democratic politics. Math. Comput. Modeling, 16(8-9)223-239

Morris, S.A., David Pratt (2003), Analysis of the Lotka -Volterra competition equations as a technological substitution model. Technological forecasting and change,70: 103-133. 